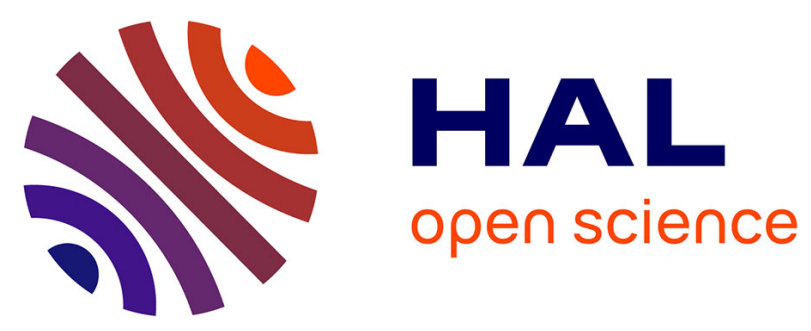

\title{
Editorial of the Special Issue: Adaptive and Intelligent Systems (AIS) for Learning, Control and Optimization in Dynamic Environments
}

\author{
Anthony Fleury, Edwin Lughofer, Moamar Sayed Mouchaweh
}

\section{- To cite this version:}

Anthony Fleury, Edwin Lughofer, Moamar Sayed Mouchaweh. Editorial of the Special Issue: Adaptive and Intelligent Systems (AIS) for Learning, Control and Optimization in Dynamic Environments. Evolving Systems, 2017. hal-01855143

\section{HAL Id: hal-01855143 \\ https://hal.science/hal-01855143}

Submitted on 7 Aug 2018

HAL is a multi-disciplinary open access archive for the deposit and dissemination of scientific research documents, whether they are published or not. The documents may come from teaching and research institutions in France or abroad, or from public or private research centers.
L'archive ouverte pluridisciplinaire HAL, est destinée au dépôt et à la diffusion de documents scientifiques de niveau recherche, publiés ou non, émanant des établissements d'enseignement et de recherche français ou étrangers, des laboratoires publics ou privés. 


\title{
Editorial of the Special Issue:
}

\section{Adaptive and Intelligent Systems (AIS) for Learning, Control and Optimization in Dynamic Environments}

\author{
Guest Editors (alphabetically):
}

Anthony Fleury, IMT Lille Douai, Douai, France

Email: Anthony.Fleury@imt-lille-douai.fr

Edwin Lughofer, Department of Knowledge-Based Mathematical Systems, Johannes Kepler University Linz, Austria

Email: edwin.lughofer@jku.at

Moamar Sayed-Mouchaweh, IMT Lille Douai, Douai, France

Email: moamar.sayed-mouchaweh@imt-lille-douai.fr

An increasing number of real world applications, such as network traffic, network monitoring, power systems etc., generates non-stationary behaviors. Therefore, the model built in order to predict their dynamics, optimize their performance or control their behavior must be adapted over time.

During the last decade, the modeling based on learning in non-stationary environments has received more and more attention in the machine learning and data mining communities. This is because the requirements from practical (industrial) side induced a permanent paradigm shift from classical batch off-line learning procedures (as conducted throughout the 80ties and 90ties to build up accurate and high-performance machine learning models) to incremental, adaptive algorithms which are able to evolve the model structures, architectures and parameters fully autonomously and on the fly, typical in a single-pass manner. It is especially the case within the scope of dynamic data streaming context, changing environmental conditions or as part of large-scale problems, e.g. web mining, multi-sensor networks, sequential video analysis, Big Data or predictive maintenance in factories for the future (FoF) as part of the Industry 4.0 activities (even an important standpoint of the Horizon 2020 objective programme see https://ec.europa.eu/programmes/horizon2020/en/h2020-sections-projects). The necessity of such algorithms is also underlined by the increasing size of the databases and storages, which induces that conventional batch learning systems cannot be applied within a reasonable time frame and sufficient accuracy. Thus, a block-wise loading of the data has to be carried out, which again requires the usage of incremental and evolving learning algorithms.

Thereby the learning algorithms are either guided by a permanent update of model parameters and structures, leading to a so-called "any-time up-to-date" experience, or by occasional update cycles 
which are often explicitly selected by active and semi-supervised learning methodologies in order to keep the measurements or feedback efforts of operators at a lowest possible level (cost reduction).

The aim of this special issue is to bring recent developments in the field of adaptive and intelligent systems in non-stationary environments to a broader audience and readership, whereas the particular focus lies not only on the (adaptive, incremental) learning aspects, but also on control and optimization aspects. The two latter has been only marginally addressed so far in literature, especially optimization that is an issue which in its classical form requires multiple iterations over batch data sets in order to assure convergence. To be able perform optimization during incrementally loaded streaming data appropriately (also terms dynamic optimization), in order to not forget and outweigh older learning solutions too intensively, is still an open central challenge. Control aims at ensuring that systems execute specified or desired behavior in order to perform specific tasks for which they were designed. Because of several external perturbations as uncertainty, erroneous or incomplete measurements, unpredicted faults or the emergence of new operation modes, control strategies require to be adapted over time in order to keep the system executing desired or safe behavior and protecting it from unforseen or dangerous behavior.

Drifts in the data streams are frequently occurring, which make older relations, dependencies between input and output data (target values), adaptively learnt into the models, obsolete. Thus the model update mechanism, no matter whether used for control or optimization, has to embed some technology for an appropriate handling of drifts, such as to increase the flexibility of the models by outweighing older learned relations and concentrate more on the new ones. A comprehensive survey about available drift handling methods in literature, including the definitions of various types of drifts, detection of drifts and appropriate reactions to them, is included in this special issue.

For this issue, we received 6 submissions, all from invited authors, for their expertise and especially from getting in contact with them at the IEEE Evolving and Adaptive Intelligent Systems (IEEE EAIS) conference 2015, which have been organized by the guest editors of this special issue (Moamar SayedMouchaweh being the main conference chair, Anthony Fleury the local arrangement chair and Edwin Lughofer the publication chair). Finally, 4 of them could be accepted after a complete review process and then included in this special issue.

In what follows, we provide a short overview of the basic concepts and algorithms to be found in each of these papers:

The paper "Discussion and Review on Evolving Data Streams and Concept Drift Adapting" (Paper \#1, EVOS-D-16-00029) by the authors Imen Khamassi, Moamar Sayed-Mouchaweh, Moez Hammami and Khaled Ghédira comprehensively discusses several methods for detecting drifts and appropriately handling them during further model updates. It thereby exploits various concepts from literature, puts them into a red line and provides a nice and round overall picture about the current state-of-the-art in drift handling methods in data streams. As mentioned by the authors "The main objective of the survey is to present an ease understanding of the concept drift issues and related works, in order to help researchers from different disciplines to consider concept drift handling in their applications." It is thus placed as the first paper in this special issue, as any incremental, single-pass streaming learning approach (as discussed in the subsequent papers) can make use of the basic concepts.

The paper "A Dedicated State Space for Power System Modeling and Frequency and Unbalance Estimation" (Paper \#2, EVOS-D-16-00030) by the authors Anh Tuan Phan, Patrice Wira and Gilles 
Hermann presents a state-space representation to model power systems like electrical distribution systems. The proposed model is able to take into account all the dynamic behavior of a multiphase power system and employs an extended recursive Kalman Filter (eKF) in order to update the power quality parameters. The proposed estimation technique is thus an adaptive method able to handle the changing power system, and it is also evolving because new state-spaces may be adjoined in the eKF. Its effectiveness has been evaluated by several tests. This also has an impact on the control law embedded in the operating principles of a shunt active power filter for power quality enhancement. The new approach is successfully applied to estimate the fundamental frequency of a power system under different conditions, where it showed significantly improved results to a related well-known method.

The paper "A fuzzy approach towards inductive transfer and human-machine interface control design" (Paper \#3, EVOS-D-16-00032) by the authors Rui Azevedo Antunes, Luis Brito Palma, Fernando Coito and Herminio Duarteramos proposes a new fuzzy clustering methodology to the development of a real-time inductive transfer embedded controller. It is used for improving the operator's proficiency, under a human-in-the-loop environment relying on visual feedback information. Thus the paper on the hand contributes to the field of adaptive, evolving controllers, but on the other hand also to human-computer interaction within a dynamic environments. Thereby, the operator behavior is modelled and enhanced from a human-machine interface fuzzy classifier and assisting scheme, which uses (high speed) real-time streaming data and additional information collected from an expert user. Experimental tests were performed by different participants under a driving simulator; the new approach confirmed to significantly improve the actual driving skills of the human operators.

The paper "An Artificial Immune System for Offline Isolated Handwritten Arabic Character Recognition" (Paper \#4, EVOS-D-16-00015) by the authors Chaouki Boufenar, Mohamed Batouche and Marc Schönauer investigates the applicability of a rule-based machine learning system, inspired by the principles and processes of Artificial Immune System (AIS), in isolated handwritten arabic characters, which is embedded in a dynamic environments. The developed system is composed of three main modules: preprocessing, feature extraction and recognition, and embeds some dynamic aspects in terms of memory cells generation within AIS. The obtained results of the proposed system are promising with a classification rate of $93.25 \%$ and typically outperform most well-known classifiers from Scikit Learn Library.

At the end, we hope that this special issue sheds light on some novel ideas of dynamic learning, control and optimization aspects in non-stationary environments. In particular, we would like to gratefully acknowledge and sincerely thank all the reviewers for their insightful comments and criticism of the manuscripts. Our thanks are also dedicated to the authors for their contributions and collaboration. Finally, we are grateful to the Editor-in-chief Plamen Angelov and the editorial office assistants of Evolving Systems for their suggestive and insightful editorial comments and support during the review and production process of this special issue.

The second guest editor also acknowledges the support of the Austrian COMET-K2 programme of the Linz Center of Mechatronics (LCM), funded by the Austrian federal government and the federal state of Upper Austria. 


\section{Guest Editors}

